\title{
Predicting the eigenmodes of a cavity containing an array of circular pipes
}

\author{
Fenech, Benjamin; Jacobsen, Finn
}

Published in:

Acoustical Society of America. Journal

Link to article, DOI:

$10.1121 / 1.1836882$

Publication date:

2005

Document Version

Publisher's PDF, also known as Version of record

Link back to DTU Orbit

Citation (APA):

Fenech, B., \& Jacobsen, F. (2005). Predicting the eigenmodes of a cavity containing an array of circular pipes. Acoustical Society of America. Journal, 117(1), 63-67. https://doi.org/10.1121/1.1836882

\section{General rights}

Copyright and moral rights for the publications made accessible in the public portal are retained by the authors and/or other copyright owners and it is a condition of accessing publications that users recognise and abide by the legal requirements associated with these rights.

- Users may download and print one copy of any publication from the public portal for the purpose of private study or research.

- You may not further distribute the material or use it for any profit-making activity or commercial gain

- You may freely distribute the URL identifying the publication in the public portal

If you believe that this document breaches copyright please contact us providing details, and we will remove access to the work immediately and investigate your claim 


\title{
Predicting the eigenmodes of a cavity containing an array of circular pipes
}

\author{
Benjamin Fenech ${ }^{\text {a) }}$ and Finn Jacobsen ${ }^{\text {b) }}$ \\ Acoustic Technology, Ørsted.DTU, Technical University of Denmark, Ørsted Plads, Building 352, \\ DK-2800 Lyngby, Denmark
}

(Received 7 March 2004; revised 26 July 2004; accepted 10 September 2004)

\begin{abstract}
An array of pipes inside a cavity, as found, for example, in a shell-and-tube heat exchanger, changes the eigenfrequencies of the cavity. It can be tedious to determine the shifted eigenfrequencies with a finite-element model. Based on previous work by Meyer and Neumann, Parker proposed a simple relationship for predicting the shifted eigenfrequencies. In this paper, results obtained from this relationship are compared with eigenfrequencies obtained from very accurate finite element simulations. From the results it can be concluded that Parker's relationship gives fairly good predictions of the eigenfrequencies for the first few modes in a cavity with pipes arranged in a rectangular configuration. The predictions are not so accurate for pipes arranged in a diamond configuration, and a modified version of the relationship is suggested for this configuration. If the number of pipes in the cavity is small, the simple relationship is no longer valid. (C) 2005 Acoustical Society of America. [DOI: 10.1121/1.1836882]
\end{abstract}

PACS numbers: 43.20.Ks, 43.58.Ta [MO].

Pages: 63-67

\section{INTRODUCTION}

A shell-and-tube heat exchanger with fluid flow past an array of pipes can be a powerful source of noise and vibration. ${ }^{1,2}$ A common excitation mechanism in such a situation is vortex shedding of the fluid flow, ${ }^{3}$ caused by, for example, heat fins on the pipes. The excitation interacts with the natural acoustic modes of the shell enclosing the tubes and the vibration modes of the pipes, thus producing an acoustic and vibrational response. One of the tasks of the noise control engineer is to calculate these natural modes, and compare them with measurements in order to identify the dominant modes. In this work only the acoustic eigenmodes of the shell (or cavity) enclosing the tubes are considered.

Calculating the eigenfrequencies of a cavity is a simple task: either analytically for simple geometries, or using a finite element model for more complex geometries. However, in a shell-and-tube heat exchanger, the tubes are part of the system, and they alter the eigenvalue problem. This phenomenon has been described analytically ${ }^{4,5}$ for single obstructing objects in a cavity, in terms of scattering and diffraction effects. However, not only are the resulting integral/ differential equations of little use in practice; but the problem gets considerably more complicated when a large number of objects are considered, among other things because of multiple scattering effects. ${ }^{4,5}$

To model the complete heat exchanger (including the array of tubes) numerically can be a tedious task, due to the large number of small pipes, even though the usual regular pattern of the pipes may speed up the process. More impor-

\footnotetext{
${ }^{a)}$ Current address: Aerodynamics \& Flight Mechanics Research Group, Aeronautics and Astronautics Department, School of Engineering Sciences, University of Southampton, Southampton, SO17 1BJ, United Kingdom. Electronic mail: bfenech@gmail.com

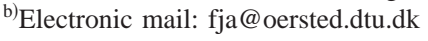

tantly, the small diameter of the pipes (compared to the outer dimensions of the shell) limits the maximum element size that can be used. This will often lead to a very large number of elements, and a corresponding demand on computational time and power.

An alternative could be to model the shell without the tubes, while adjusting some parameter to take account of the effect of the tubes. Parker has proposed a very simple expression in terms of an effective speed of sound, ${ }^{6}$ referring to the concept of added (virtual) mass as used by Meyer and Neumann. ${ }^{7}$ The underlying reasoning of this model is not explained very clearly. However, both Parker ${ }^{6}$ and Blevins ${ }^{2}$ have presented experimental results that confirm this relationship.

Our purpose in this paper is to present some numerical simulations that have been performed in order to test Parker's expression and get an idea of its accuracy and limitations. To simplify the calculations, the problem has been reduced to two dimensions by taking a perpendicular cross section along an inline shell-and-tube heat exchanger, such that the acoustic velocity is in the plane perpendicular to the pipes' axes. Moreover, no external fluid flow is considered.

\section{THE EFFECTIVE SPEED OF SOUND}

The concept of an effective speed of sound was originally proposed by Meyer and Neumann, who developed a relationship to predict the reduced speed of sound in an "acoustic lens.", They stated that an alternating flow around an array of fixed, rigid objects has an increased inertia, in the same way as an oscillating object in a fluid has an added (virtual) mass. The added mass or inertia depends in both cases on the shape of the rigid object; for a circular cylinder in unrestricted two-dimensional (2-D) flow, the added mass equals the mass of the fluid displaced by the cylinder. For an array of circular pipes, the relationship becomes ${ }^{6}$ 


$$
\frac{c_{e}}{c}=\frac{1}{\sqrt{1+\sigma}},
$$

where $c_{e}$ is the effective speed of sound in the cavity with the pipes, $c$ is the speed of sound, and $\sigma$ is the volume fraction, i.e., the percentage of the volume occupied by the pipes. Note that this relationship is only valid for pipes of circular cross section. In formulating this relationship, it was assumed that the compressibility of the fluid does not change when an array of acoustically hard objects is introduced. This assumption is questionable, especially when applied to modes in a cavity, where an object can sit in a region of no flow (pressure maxima). In this case, it is more likely that the presence of the object increases the stiffness, rather than the mass of the fluid, as suggested by Morse and Ingard. ${ }^{4}$ Another factor not taken into account is that, since the added mass depends on the flow pattern, it is affected by other nearby boundaries $\left(\right.$ Massey $\left.^{8}\right)$.

\section{RESULTS}

The simplified problem has been modeled as a number of circular bodies in a rectangular cavity. All the boundaries were treated as rigid. The numerical calculations were done using the eigenvalue solver of MSC Actran, a commercial finite element package. This solver uses a block Lanczos method with a Sturm sequence check, and uses Actran's sparse solver as an internal linear solver. ${ }^{9}$ Triangular quadratic elements were used. A number of pretests showed that an element size equal to the radius of the pipes gave sufficiently accurate results, even though geometrically the modeled bodies were not perfectly circular. With such an element size, the number of elements per wavelength was at least five in the frequency range of interest. All post-processing was done using Matlab.

Two different, widely used pipe configurations were examined: a rectangular and a diamond configuration; see Fig. 1. The geometries shown are fixed in such a way as to reduce the number of variables of the problem. The simulations were done in terms of four nondimensional parameters: the volume fraction $(\sigma)$, the cavity aspect ratio $(a / b)$, the largest number of pipes in the $x$ direction $\left(n_{x}\right)$, and the normalized eigenfrequencies of the system $(\mathrm{fa} / \mathrm{c})$.

\section{A. Number of pipes}

Equation (1) applies for an array of pipes, and should not depend on the number of objects in the array. This was checked in the first simulation. The cavity aspect ratio $a / b$, pipe spacing $d$, and volume fraction $\sigma$ were all fixed, while the number of pipes in the cavity was varied. This was done for the two different pipe configurations, and for three different volume fractions: $3 \%, 12.5 \%$, and $50 \%$. For a fixed volume fraction, the cavity dimensions increase as the number of pipes increases. Figure 2 shows a plot for a rectangular configuration of pipes with a volume fraction of approximately $50 \%$. Each curve represents a particular mode of the first 50 modes, sorted by increasing eigenfrequencies.

The plot can be divided into two regions: one where the eigenfrequencies depend on the number of pipes in the cav-

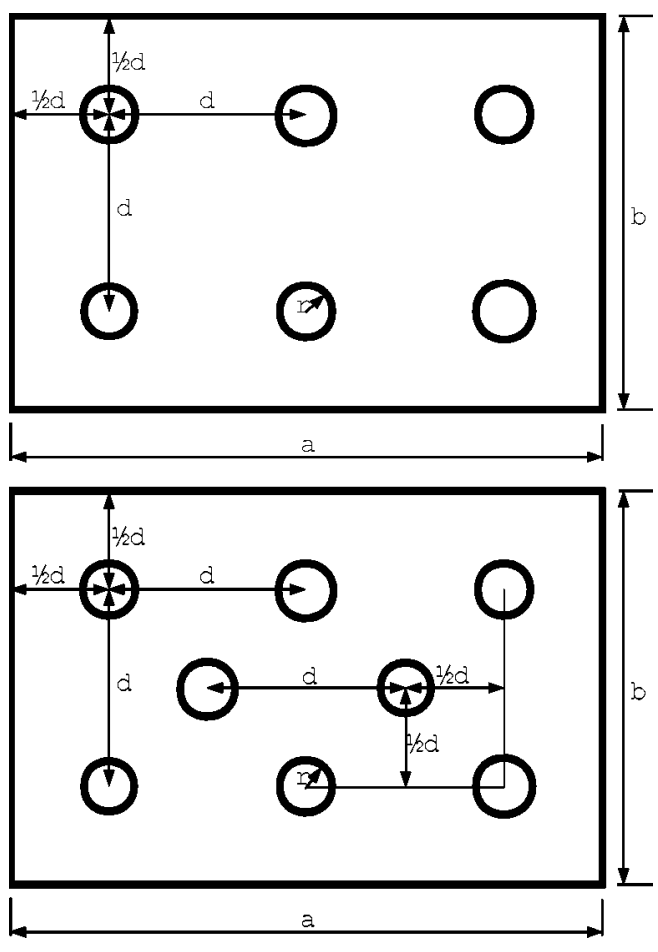

FIG. 1. Cross section of the heat exchanger. (a) Rectangular configuration. (b) Diamond configuration.

ity, and another one where the normalized eigenfrequencies are almost independent of the number of pipes in the cavity. The division occurs between $n_{x}=10$ and $n_{x}=15$. The same trend has been observed for a diamond configuration of pipes (not shown). For smaller volume fractions, the dependence of the eigenfrequencies is less pronounced; for $\sigma=3 \%$, practically all the modes are represented by horizontal lines along the whole abscissa.

\section{B. Volume fraction}

The horizontal curves shown in Fig. 2 for a large number of pipes suggest the existence of a simple relationship for

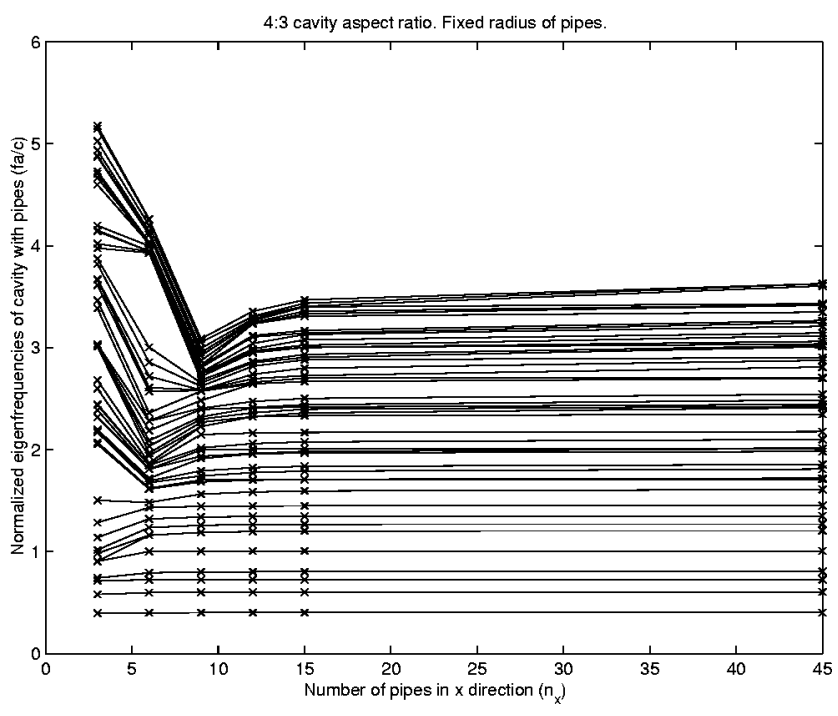

FIG. 2. Effect of the number of pipes on the modes of a cavity. Rectangular configuration. The volume fraction was approximately $50 \%$. 

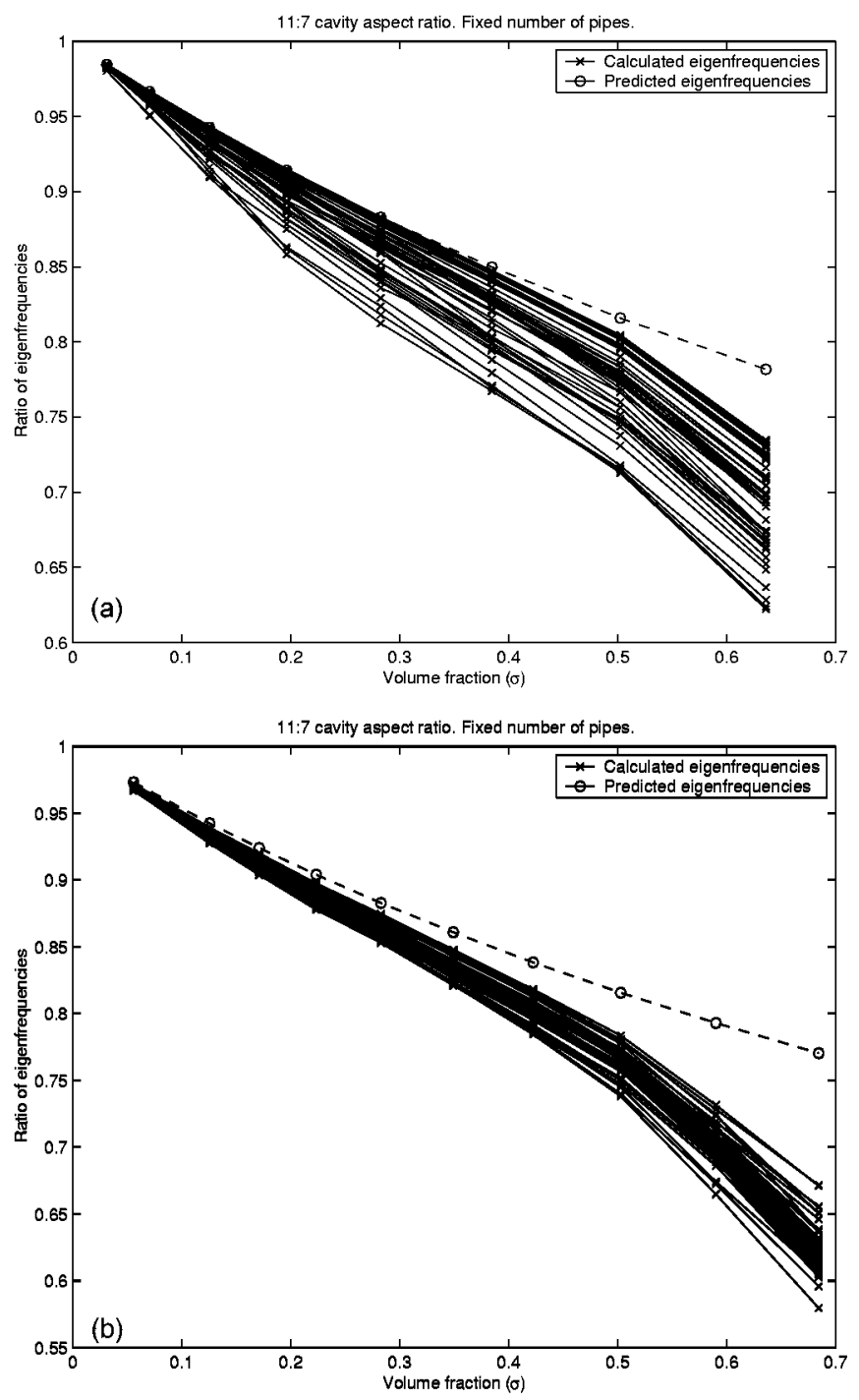

FIG. 3. The ratio of eigenfrequencies as a function of the volume fraction: calculated (ACTRAN) and predicted [using Eq. (2)]. (a) Rectangular configuration. (b) Diamond configuration.

predicting the shifting of eigenfrequencies. An additional set of simulations were thus carried out to test the accuracy of Eq. (1). For these simulations, the number of pipes in the cavity was kept constant, whereas the volume fraction was varied. A similar, but slightly modified cavity aspect ratio of 11:7 was used in the subsequent tests to avoid modal degeneracy for the first 50 modes.

Equation (1) can be rewritten in terms of the eigenfrequencies of the cavity with and without the pipes,

$$
\frac{f_{m, n \text { pipes }}}{f_{m, n \text { nopipes }}}=\frac{1}{\sqrt{1+\sigma}} .
$$

The eigenfrequencies of the cavity without pipes, $f_{m, n \text { nopipes }}$, can be calculated from

$$
f_{m, n \text { nopipes }}=\frac{c}{2} \sqrt{\left(\frac{m}{a}\right)^{2}+\left(\frac{n}{b}\right)^{2}}, \quad m, n=0,1,2,3, \ldots .
$$

A significant number of eigenfrequencies was generated using Eq. (3), and sorted in ascending order, and the first 50 were compared with the eigenfrequencies obtained from the
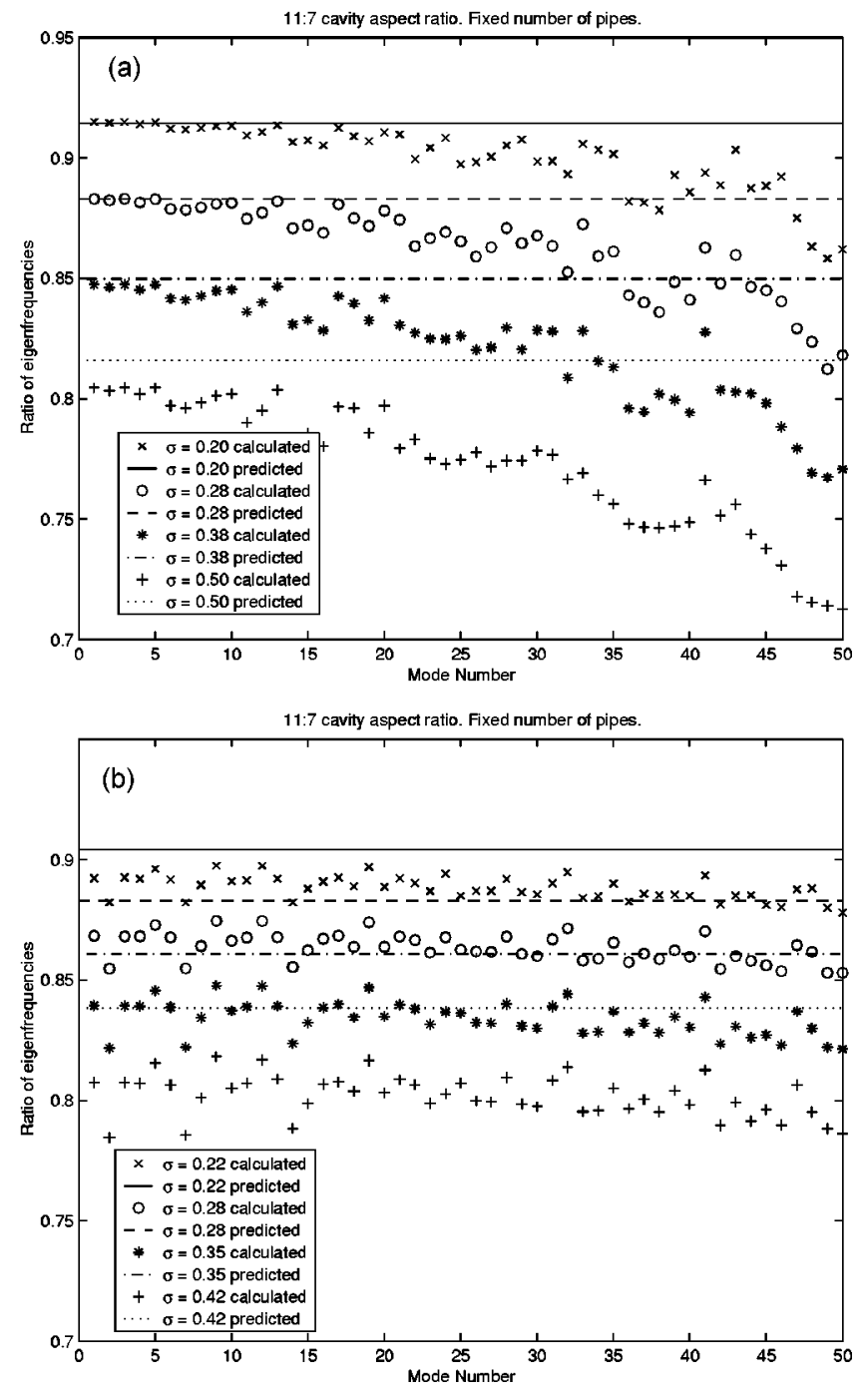

FIG. 4. The ratio of eigenfrequencies as a function of the mode number: calculated (ACTRAN) and predicted [using Eq. (2)]. (a) Rectangular configuration. (b) Diamond configuration.

numerical simulations to obtain the ratio $f_{m, n \text { pipes }} / f_{m, n \text { nopipes }}$. Eight different volume fractions were tested for both a rectangular and a diamond pipe configuration; see Figs. 3(a) and 3(b). The figures also show the curve corresponding to Eq. (2).

According to the numerical simulations, an array of pipes decreases the eigenfrequencies of a cavity, and the effect increases as the volume fraction occupied by the pipes grows. For a rectangular array of pipes with a volume fraction of up to approximately 35\%, the relationship suggested by Parker compares fairly well with the upper curves in the plot. These curves correspond to the first few modes (this can be verified in Fig. 4, which is described in the next paragraph). For higher volume fractions, the eigenfrequencies calculated numerically are somewhat lower than predicted. The relationship fails to predict the behavior of higher-order modes, which deviate gradually from the behavior of the first few modes. A diamond configuration of pipes shows the same trends, except that the relationship is less accurate in predicting the shifting of the eigenfrequencies, even for small volume fractions. 
(a)

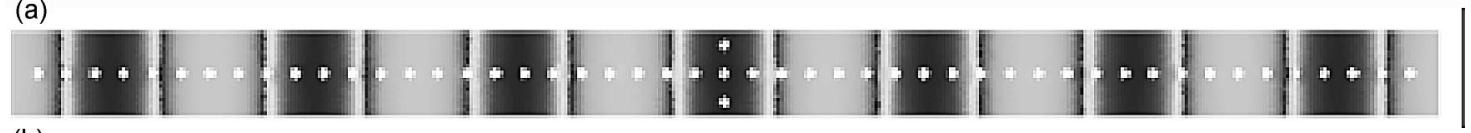

(b)

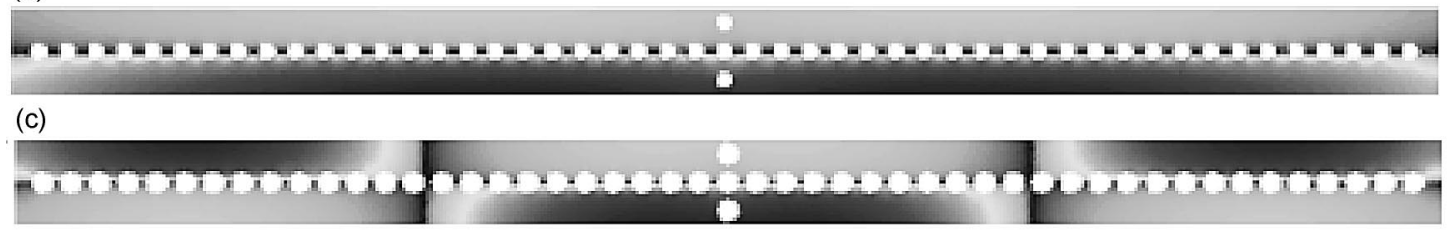

FIG. 5. Mode order shifting. The 14th mode (modes sorted by increasing eigenfrequencies) in the same cavity, with the same configuration of pipes. The mode shape is different for different radii. (a) Radius $=0.02 \mathrm{~m}$. Mode $(14,0)$. (b) Radius $=0.03 \mathrm{~m}$. Mode $(0,1)$. (c) Radius $=0.04 \mathrm{~m}$. Mode $(2,1)$.

The accuracy of the predictions for higher-order modes is illustrated in Fig. 4, which shows the ratio of eigenfrequencies as a function of the mode number for four different volume fractions; those are considered to be most relevant in practice. For a given volume fraction, the predicted frequency ratio is represented by a horizontal curve. For a rectangular configuration of pipes with volume fractions of $20 \%$, $28 \%$, and $38 \%$, the predicted frequency ratio compares very well with the numerical calculations for the first ten modes. For higher-order modes, the deviations from predicted values are clearly observable. The plot for a diamond configuration of pipes differs in two aspects: first, the predictions are less accurate even for the first few modes at small volume fractions; second, the calculated eigenfrequencies do not "roll off" appreciably for higher-order modes. A further characteristic, which is present in both plots, is that different modes are grouped together, and these patterns persist for different volume fractions. This suggests that the shift of eigenfrequencies is also a function of the mode shape, as stated, for example, by Morse and Ingard. ${ }^{4}$

\section{DISCUSSION}

From Fig. 2 it is clear that Eq. (2) is not valid for the general case of a number of rigid objects in a rigid cavity. For a small number of pipes, the shift in eigenfrequencies is dependent on the number of pipes. This dependence can be explained by referring to a simplified approximate relationship for the shift of eigenfrequencies by a rigid scattering object developed by Morse and Ingard, ${ }^{4}$

$$
K_{N}^{2} \approx \eta_{N}^{2}-\frac{k^{2} \sigma}{\Lambda_{N}}\left[\left\langle\rho^{2} c^{2} U_{N}^{2}\right\rangle-\left\langle\phi_{N}^{2}\right\rangle\right]
$$

where $K_{N}^{2}$ are the eigenfrequencies of the cavity with the scattering object, $\eta_{N}^{2}$ are the eigenfrequencies of the empty cavity, $\left\langle U_{N}^{2}\right\rangle$ is the spatially averaged mean square velocity amplitude, $\left\langle\phi_{N}^{2}\right\rangle$ is the spatially averaged mean square pressure amplitude, and $\Lambda_{N}$ is a normalization constant. The "new" eigenfrequencies depend on two correction terms: one decreasing and the other one increasing the "old" eigenfrequencies, depending on where the object is sitting, i.e., near a velocity or a pressure node. This prediction has been validated by modeling a cavity with a single line of pipes: the eigenfrequencies were increased when the pipes sat on a velocity node, and decreased when the pipes were on a pressure node. The fact that this up-down behavior breaks down for a large number of pipes may be explained by looking at the mode shapes: the pipes are almost always sitting on a pressure node, which makes the second correction term negligible. A line of pipes seems to either "pull" a nodal line, or force some particular modes (having nodal lines along the existing line of pipes) to occur at a much lower frequency, thus changing the mode ordering completely. This effect can be seen in Figs. 5(a)-5(c), which show the 14th mode in a particular cavity having the same configuration but with different pipe diameters. For the smallest diameter [Fig. 5(a)], the 14th mode corresponds to $(14,0)$, the same as one would predict for an empty cavity. However, if the diameter is increased by a factor of 1.5, shown in Fig. 5(b), mode $(0,1)$ occurs at a lower frequency than mode $(14,0)$ and becomes the 14th mode. When the initial diameter is doubled, even mode $(2,1)$ occurs at a lower frequency than mode $(14,0)$, as shown in Fig. 5(c).

For a rectangular array of pipes, Eq. (2) gives better predictions for the first few modes than for higher-order modes. This is, in general, acceptable, since, in practice, the first modes are usually the most troublesome. However, the same relationship is not good for a diamond configuration. In formulating Eq. (1), it was assumed that adjacent pipes do not affect the flow pattern, so that a simple relationship for the added mass could be used. In a diamond configuration, the effect of adjacent pipes on the flow pattern might be more significant than in a rectangular configuration. If this is the case, a corrected value of the added mass for pipes arranged in a diamond configuration can yield a better prediction expression than Eq. (2). Such a correction can be determined either experimentally or perhaps analytically. In this project, a simpler approach was taken. Referring to Fig. 3, Eq. (2) gives an underestimation of the ratio of eigenfrequencies. Thus, as an alternative relationship, the reciprocal with a changed sign was considered, i.e., $f_{m, n \text { pipes }} / f_{m, n \text { nopipes }}$ $=\sqrt{1-\sigma}$. It turned out that, for a diamond configuration of pipes, the data fell in between the two relationships. It was concluded that, for such a configuration, a weighted combination of the two equations,

$$
\frac{f_{m, n \text { ipes }}}{f_{m, n \text { nopipes }}}=\frac{2 \times \sqrt{1-\sigma}}{5}+\frac{3}{5 \times \sqrt{1+\sigma}},
$$

gives more accurate predictions than Eq. (2). This result is a purely empirical one. Figure 6 shows the improved predic- 
11.7 cavity aspect ratio. Fixed number of pipes.

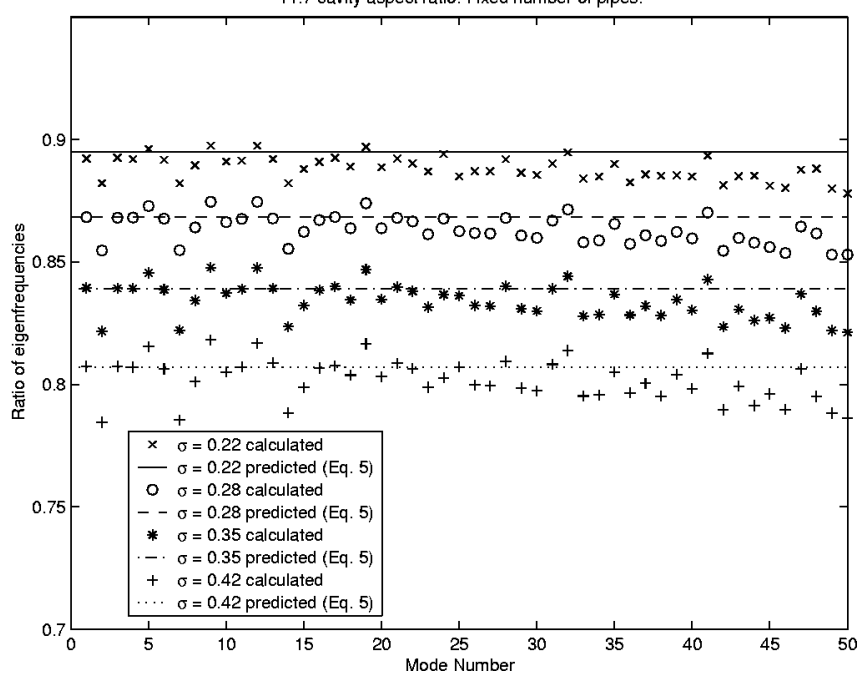

FIG. 6. Predictions using Eq. (5) compared to ACTRAN simulations, for a diamond configuration of pipes.

tions of eigenvalues for a cavity with a diamond configuration using Eq. (5) [compare with Fig. 4(b)].

In both configurations, higher-order modes deviate from the behavior of the first few modes. This is more significant for the rectangular configuration. The most likely reason for this is the change in mode ordering. Whenever a change in the mode ordering occurs, the ratio of eigenfrequencies $f_{m, n \text { pipes }} / f_{m, n \text { nopipes }}$ becomes invalid. Furthermore, even if the ratio is corrected so that the two frequencies correspond to the same mode shape, it still deviates considerably from the predicted ratio. Further tests have shown that changes in mode ordering are less likely to occur with a diamond configuration, thus explaining the collapsed curves for this configuration in Fig. 3.

Further simulations have been carried out, including cavities with irregularly spaced pipes, and tests for a cavity having an aspect ratio of 37:5. The results were similar to those presented here, and are therefore not included.

\section{CONCLUSIONS}

An array of acoustically rigid pipes affects the eigenfunctions and eigenvalues of a cavity. For a sufficiently large number of pipes, the eigenfrequencies are decreased, pro- vided that the pipes are not intentionally placed at velocity nodes. The reduction depends on the volume fraction occupied by the pipes. Fairly good predictions of the magnitude of these reductions are possible using a very simple relationship having only one variable: the volume fraction. This relationship is reasonably accurate for the first few modes (say the first ten, sorted by increasing eigenfrequencies), for a rectangular configuration of pipes having volume fractions up to around $35 \%$. With a diamond configuration of pipes, more accurate results can be obtained by using a slightly different, empirically derived relationship.

For higher-order modes, the fact that the order of the modes is likely to change means that the validity of the simple expression breaks down. This drawback is not so pronounced for a diamond configuration of pipes, and for cavities with a high aspect ratio. Furthermore, this phenomenon makes Eq. (2) useless for predicting the eigenfrequencies of a cavity with only a single line of pipes present.

Finally, pressure nodal lines tend to occur along lines of pipes. This can have implications on noise abatement techniques used in such applications.

\section{ACKNOWLEDGMENTS}

This work has been carried out in collaboration with the Danish company Ødegaard \& Danneskiold-Samsøe A/S. The authors acknowledge the significant contribution from Graeme Keith to all the stages of the project.

${ }^{1}$ R. D. Blevins, "The effect of sound on vortex shedding from cylinders," J. Fluid Mech. 161, 217-237 (1985).

${ }^{2}$ R. D. Blevins, Flow-Induced Vibration, 2nd ed. (Krieger Publishing, Florida, 1994).

${ }^{3}$ M. S. Howe, Acoustics of Fluid-Structure Interactions (Cambridge University Press, Cambridge, 1998).

${ }^{4}$ P. M. Morse and K. U. Ingard, Theoretical Acoustics (McGraw-Hill, New York, 1968).

${ }^{5}$ D. Ouellet, J.-L. Guyader, and J. Nicolas, "Sound field in a rectangular cavity in the presence of a thin, flexible obstacle by the integral equation method," J. Acoust. Soc. Am. 89, 2131-2139 (1991).

${ }^{6}$ R. Parker, "Acoustic resonances in passages containing banks of heat exchanger tubes," J. Sound Vib. 57, 245-260 (1978).

${ }^{7}$ E. Meyer and E. G. Neumann, Physical and Applied Acoustics (Academic, New York, 1972).

${ }^{8}$ B. Massey and J. Ward-Smith, Mechanics of Fluids, 7th ed. (Stanley Thornes Publishers Ltd., City, 1998).

${ }^{9}$ Free Field Technologies, MSC, ACTRAN 2003 User's Guide, 2002. 